Article original

\title{
Extraction et analyse de la phase aqueuse de l'emmental à 4 stades d'affinage
}

\author{
D Salvat-Brunaud, JL Maubois, Y Le Graët, M Piot, MB Maillard, \\ C Corre, A Thierry
}

Laboratoire de recherches de technologie laitière, INRA, 65, rue de Saint-Brieuc, 35042 Rennes cedex, France

(Reçu le 17 janvier 1995 ; accepté le 22 février 1995)

\begin{abstract}
Résumé - Grâce à la mise au point d'un équipement prototype de pressage, une amélioration de la méthodologie d'extraction de la phase aqueuse des fromages proposée initialement par Barthel et al (1928a) a été réalisée. Les phases aqueuses d'emmental à 4 stades d'affinage : sortie saumure, entrée et sortie cave chaude, commercialisation, ont été extraites et caractérisées sur les plans bactériologique, physico-chimique et biochimique. La teneur en substance sèche initialement de $90 \mathrm{~g} / \mathrm{kg}$ de phase aqueuse atteint $170 \mathrm{~g} / \mathrm{kg}$ dans le fromage affiné. Cet accroissement découle de l'élévation (multiplication par 5) de la teneur en azote solubilisé du fait de la protéolyse, notamment lors de l'affinage en cave chaude ; $56 \%$ de l'azote total déterminé dans le fromage affiné sont sous la forme d'acides aminés libres. L'évolution des teneurs en sucre et en acides organiques est conforme à leur métabolisation par les bactéries lactiques et propioniques (consommation de $25 \%$ du lactate majoritairement de la forme $\mathrm{L}$ ). L'analyse des formes minérales montre une évolution considérable des sels phosphocalciques par rapport au lait : rapport $\mathrm{Ca} / \mathrm{P}$ variant entre 8,2 et 5,4 au lieu de 1,3 . Les teneurs en $\mathrm{Na}$ et $\mathrm{Cl}$ ne sont pas en accord avec leurs proportions dans la molécule de $\mathrm{NaCl}$, ce qui conduit à penser qu'une partie de l'ion $\mathrm{Cl}$ est mobilisé dans d'autres formes salines du type chlorhydrate. La caractérisation de la phase aqueuse des fromages apparaît au travers de cette première étude comme une méthodologie privilégiée de suivi de la fabrication et de l'affinage des fromages.
\end{abstract}

emmental / phase aqueuse / affinage / composition / presse hydraulique

Summary - Extraction and composition of the aqueous phase of Emmental during ripening. $A$ new pressing unit was designed to improve the extraction methodology of the cheese aqueous phase, originally proposed by Barthel et al (1928a). Emmental aqueous phases extracted from cheeses at different ripening ages: after brining - before and after the warm room and at commercialization were characterized bacteriologically, physico-chemically and biochemically. Total solids content which is originally $90 \mathrm{~g} / \mathrm{kg}$ of the aqueous phase increases up to $170 \mathrm{~g} / \mathrm{kg}$ in the fully ripened cheese phase. Most of this increase in caused by the proteolysis, mainly during the warm room period, which leads to a 5 times higher nitrogen content. A large part (56\%) of the nitrogen water soluble components is free amino acids, some being at oversaturation (Tyr and Glu). No residual sugar was determined in aqueous phase and $25 \%$ of the lactate present in the brined cheese phase is consumed by propionibacteria during the warm room period, mainly as $L$ form. Deep modifications of phosphocalcic salts are detected: Ca/P ratio varies between 8.2 and 5.4 in the aqueous phase instead of 1.3 in milk. $\mathrm{Na} / \mathrm{Cl}$ ratio also does not agree with the theoretical value in $\mathrm{NaCl}$ molecule, leading to the conclusion that part of Clion is mobilized in other saline forms, maybe chlorhydrate. Characterization of the cheese 
aqueous phase appears to be a privileged methodology to follow precisely the effects of technological cheesemaking parameters and ripening conditions.

\section{Emmental / aqueous phase / ripening / composition / hydraulic press}

\section{INTRODUCTION}

Le fromage peut être identifié à un système biphasique comprenant un réseau solide poreux de paracaséine adsorbant la matière grasse et une phase aqueuse : le lactosérum de fin d'égouttage s'enrichissant en sel au cours de l'opération de salage (Maubois et Mocquot, 1971 ; Morris et al, 1988). Pendant l'affinage, les colonies de bactéries lactiques et non lactiques situées à l'interface réseau phase aqueuse (Favrot, 1994) interviennent par leurs enzymes pariétales (cellules intactes) et intracellulaires (cellules lysées) sur la dégradation de la paracaséine et de certains composants de la phase aqueuse. Les enzymes endogènes du lait (plasmine, lipase) ou ajoutées (présure) participent également à cette évolution de structure et de composition des 2 phases du bioréacteurfromage.

La phase aqueuse est donc le milieu dans lequel croissent les micro-organismes composant l'écosystème intervenant pendant l'affinage et dans lequel s'accumulent les produits hydrosolubles résultant du métabolisme bactérien et des réactions enzymatiques, produits à l'origine des molécules odorantes et sapides (Adda, 1987). II découle de cette constatation qu'une caractérisation biochimique et physico-chimique approfondie de cette phase aqueuse aux différents stades d'affinage, en complément de l'analyse du fromage correspondant, constituerait une méthodologie puissante et riche d'enseignements sur les mécanismes de l'affinage.

L'extraction de la phase aqueuse par soumission d'un mélange fromage râpé sable à une forte pression mécanique a été proposée dès 1928 par Barthel et al (a) comme moyen privilégié pour « suivre la marche de la maturation " et " l'action de la présure sur la maturation ". L'extraction par l'eau des composants solubles du fromage étaít déjà critiquée par ces auteurs car susceptible de modifier profondément les équilibres physico-chimiques et de conduire ainsi à des résultats erronés sur les formes solubles de l'azote. L'application de l'extraction par pression à différents types de fromages à pâte dure a permis de démontrer l'absence d'activité coagulante due à la présure dans le jus d'emmental (Barthel et al, 1928b) et de suivre l'accroissement des teneurs en azote soluble au cours de l'affinage de fromages de lait écrémé (Sandberg et al, 1930). Les teneurs en azote des jus extraits de fromages commerciaux tels que emmental, édam, roquefort, limbourg et stilton ont été mesurées (Sandberg et al, 1930), ainsi que l'évolution des teneurs i) en lactose et en acide lactique du cheddar (Mc Dowall et Dolby, 1936), ii) en $\mathrm{NaCl}$, azote, $\mathrm{Ca}$ et $\mathrm{P}$ de l'edam en cours d'affinage (Monib, 1962 cité par Morris et al, 1988).

La méthodologie proposée par Barthel et al (1928a) a été plus récemment reprise par Morris et al (1988), à l'aide d'un équipement de pressage modernisé dans le but d'étudier la composition en minéraux et en acides aminés libres d'un fromage cheddar âgé d'un mois. Enfin, Lucey et al (1993) ont utilisé un équipement identique et cette méthodologie pour l'étude comparée du pouvoir tampon du fromage emmental entier et de son « jus d'extraction " à différents stades d'affinage.

L'équipement développé par Morris et al (1988) ne permettant l'obtention que de $30 \mathrm{ml}$ de jus environ par pressage, ce qui limitait d'autant l'ampleur des analyses pouvant être réalisées, nous nous sommes attachés dans la présente étude à concevoir les 
éléments d'une presse hydraulique propre à traiter 6 fois plus de fromage. Ce matériel a été ensuite utilisé selon un protocole optimisé pour l'extraction des phases aqueuses de fromages emmental prélevés dans la même entreprise à quatre stades d'affinage. Ces phases aqueuses ont été caractérisées sur les plans bactériologique, physico-chimique et biochimique.

\section{MATÉRIELS ET MÉTHODES}

\section{Échantillons d'emmental}

Des meules d'emmental français ont été prélevées dans une entreprise laitière (Unicopa, $\mathrm{Ca}$ rhaix, France) à 4 stades d'affinage : une meule en sortie de saumure (identifiée comme EmA), une meule en entrée de cave chaude $(E m B)$, une meule en sortie de cave chaude (EmC) et une portion d'emmental affiné prêt à la commercialisation $(E m D)$. Les fromages étaient découpés en secteurs de 2 à $2,5 \mathrm{~kg}$, constituant des échantillons représentatifs de la meule. Chaque secteur était emballé individuellement sous vide et stocké congeléà $-20^{\circ} \mathrm{C}$. Avant pressage, chaque secteur ètait décongelè pendant 15 à $20 \mathrm{~h}$ à $4^{\circ} \mathrm{C}$.

Les phases aqueuses correspondant respectivement aux 4 emmentals, $\mathrm{EmA}, \mathrm{EmB}, \mathrm{EmC}$ et EmD ont été identifiées par les lettres A, B, C et D.

Pour chacun des 4 fromages, 3 échantillons de $1,8 \mathrm{~kg}$ ont été pressés. Les résultats présentés dans cette étude pour les phases aqueuses A, B, $C$ et $D$ sont les moyennes des analyses pratiquées sur les trois phases obtenues pour chaque stade d'affinage.

\section{Protocole d'extraction de phase aqueuse d'emmental}

Un secteur d'emmental était finement râpé après élimination de la croûte et d'une zone de 0,3 à $0,5 \mathrm{~cm} ; 1800 \mathrm{~g}$ de fromage râpé étaient mélangés à $3600 \mathrm{~g}$ de sable de Fontainebleau de granulométrie comprise entre 150 et $250 \mu \mathrm{m}$ (Prolabo, France).

Le mélange était placé, au sein d'une toile jetable (disposable cheddar cheese cloth, Smith and Nephew Extruded Films Ltd, Hull, Royaume-
Uni), dans un moule en acier inoxydable dont la partie interne perforée permet l'écoulement du jus (Perfora type 5, APV Baker, Evreux, France).

Le moule rempli du mélange sable-fromage ètait placé sur une plaque en acier inoxydable (pièce prototype usinée par Mecalef Industrie, Rennes, France) dont la fonction est double : recueillir la phase aqueuse et une fraction lipidique dans une rainure qui débouche sur une gouttière et éviter la déformation du moule sous l'effet de la pression exercée par le piston du vérin.

Le mélange était pressé à température ambiante à l'aide d'une presse hydraulique électrique (presse prototype réalisée par Trans Hydro, Morlaix, France). La montée en pression était réalisée lentement selon une cinétique définie après plusieurs essais préliminaires (fig 1) : montée de la pression atmosphérique à 5 bars pendant les 10 premières minutes, montée de 5 à 37 bars en 100 min, montée de 37 à 42 bars en 40 min puis maintien de la pression à 42 bars pendant $30 \mathrm{~min}$. Au cours des $3 \mathrm{~h}$ de pressage, la fraction lipidique liquide jaune, et la phase aqueuse diaphane étaient recueillies dans une éprouvette (fig 2).

Le pressage terminé, l'éprouvette était refroidie à $4^{\circ} \mathrm{C}$ pendant $2 \mathrm{~h}$.

\section{Préparation des phases aqueuses avant analyse}

La matière grasse sumageante, solidifiée lors du refroidissement à $4^{\circ} \mathrm{C}$, était perforée en deux points pour permettre l'écoulement de la phase aqueuse.

Une partie de la phase aqueuse était aussitôt prélevée pour les numérations bactériennes. Le reste de la phase aqueuse était, pour cette étude, pré-filtré puis filtré sur membranes en acétate de cellulose de seuils de coupure respectifs de 1,2 et $0,45 \mu \mathrm{m}$ (Sartorius, Palaiseau, France). La phase aqueuse ainsi préparée était stockée à $-20^{\circ} \mathrm{C}$, puis décongelée en chambre froide $\left(4^{\circ} \mathrm{C}\right)$ avant analyse.

\section{Numérations bactériennes}

Les dilutions nécessaires étaient effectuées dans de l'eau peptonée salée à $1 \mathrm{~g} / \mathrm{l}$ de Tryptone (Biokar, Beauvais, France) et $8,5 \mathrm{~g} / \mathrm{l} \mathrm{NaCl}$ (Merck, Nogent-sur-Marne, France). 


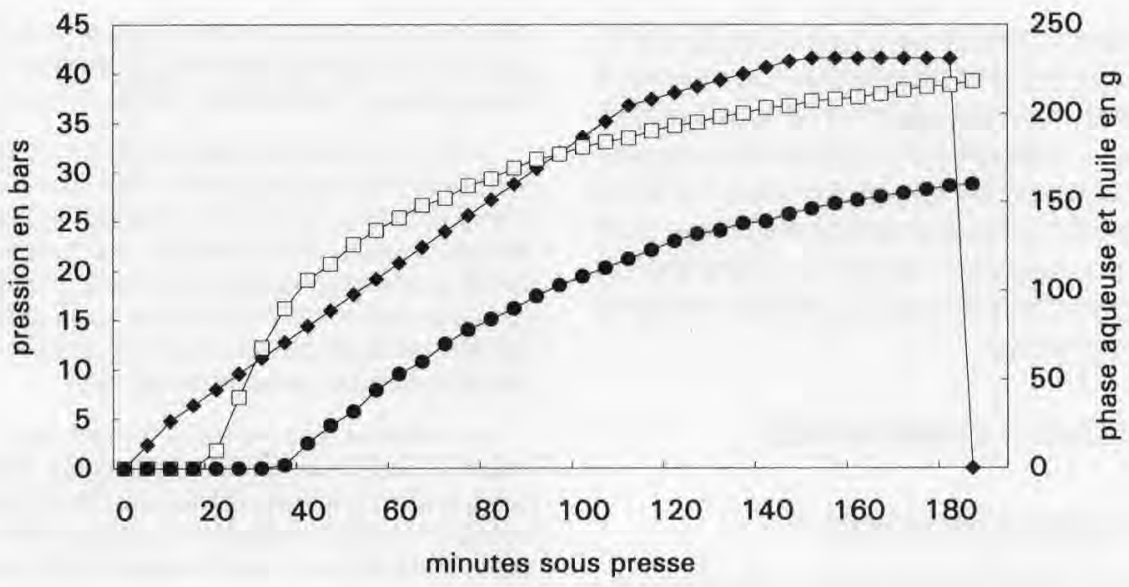

Fig 1. Cinétiques de montée en pression et d'écoulement de l'huile et de la phase aqueuse de $1,8 \mathrm{~kg}$ d'emmental. Pression (bar) exercée sur le mélange emmental-sable ; $\square$ huile d'emmental (g) ; - phase aqueuse d'emmental (g).

Pressure kinetic, oil and aqueous phase collected from $1.8 \mathrm{~kg}$ emmental cheese. Pressure (bar) applied on the cheese-sand mixture; $\square$ oil $(g)$; aqueous phase $(g)$.

La flore mésophile était dénombrée sur Plate Count Agar (PCA) (Biokar, Beauvais, France) après incubation 3 jours à $30^{\circ} \mathrm{C}$.

Les coliformes totaux étaient recherchés sur Violet Red Bile Agar (VRBA) (Difco, Detroit, ÉtatsUnis) après incubation 2 jours à $30^{\circ} \mathrm{C}$.

Les dénombrements de bactéries propioniques étaient effectués sur le milieu Propiobac (Standa Industrie, Caen, France) développé par Madec et al (1994). Les boîtes de Petri étaient incubées en anaérobiose (Anaerocult, Merck, Nogent-sur-Marne, France) à $30^{\circ} \mathrm{C}$ pendant 6 jours.

\section{Analyses physico-chimiques}

Le $\mathrm{pH}$ était mesuré à l'aide d'un $\mathrm{pH}$-mètre (PHM 84 Research, Radiometer, Copenhague, Danemark). La matière grasse des fromages était dosée par la méthode acido-butyrométrique de Gerber. Les teneurs en matière sèche étaient déterminées par dessiccation à l'étuve $\left(102-105^{\circ} \mathrm{C}\right)$ pendant $7 \mathrm{~h}$.

Le lactose, le glucose, le galactose et les acides lactique, acétique, propionique et butyrique étaient dosés par chromatographie liquide haute performance sur une colonne de $7,5 \mathrm{~mm}$ de diamètre sur $300 \mathrm{~mm}$ de longueur contenant la résine Aminex A6 (Biorad, Richmond, États-Unis), résine de type sulfonique : groupement $-\mathrm{SO}_{3} \mathrm{H}^{+}$ fixé, réticulation de $8 \%$, avec $\mathrm{H}_{2} \mathrm{SO}_{4} 0,01 \mathrm{~N}$ comme solvant et un débit de $1 \mathrm{ml} / \mathrm{min}$ à température ambiante. $L$ 'isomère $L(+)$ du lactate était dosé par méthode enzymatique (Boehringer Mannheim, Meylan, France).

Les cendres étaient déterminées en double, par incinération de la matière sèche dans un four à $550^{\circ} \mathrm{C}$ pendant $3 \mathrm{~h} 30$ à partir de $2 \mathrm{~g}$ pour les fromages et de $5 \mathrm{~g}$ pour les phases aqueuses. Le chlore était dosé par titrimétrie (Chloride Analyzer 926, Ciba-Corning, Halsteard, Royaume-Uni). Le dosage du phosphore était effectué selon la méthode préconisée par la FIL (1987). Les déterminations des teneurs en calcium, magnésium, potassium, fer, cuivre, manganèse et sodium, étaient réalisées par spectrométrie d'absorption atomique sur un appareil Varian AA 300.

Les dosages d'azote (exprimés en matière azotée $\mathrm{N} \times 6,38$ ) étaient réalisés par la méthode de Kjeldhal : azote total (NT) sur la phase aqueuse, azote non caséinique (NCN) sur la fraction soluble à $\mathrm{pH} 4,6$ et azote non protéique (NPN) sur la fraction soluble dans l'acide trichlo- 


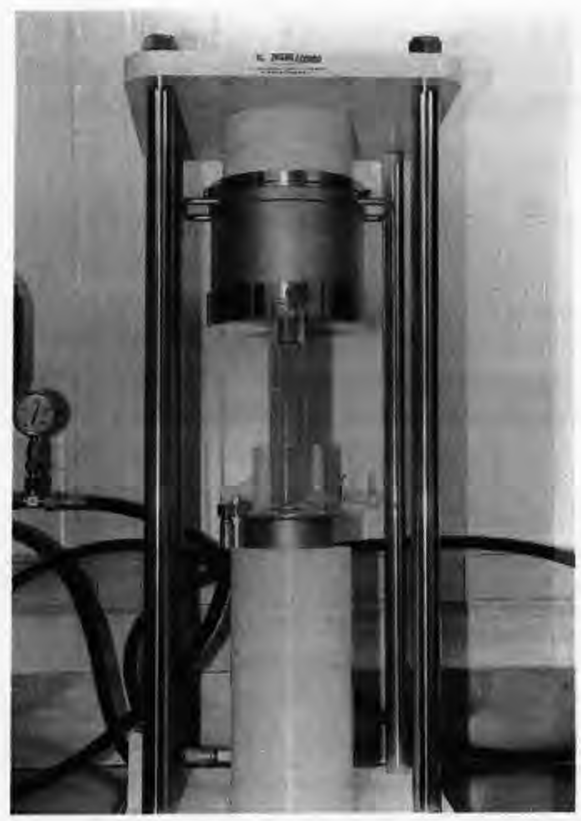

Fig 2. Obtention de phase aqueuse et d'huile d'emmental par pressage d'un mélange emmental-sable à l'aide d'une presse hydraulique électrique.

Emmental aqueous phase and oil being pressed from the sand and cheese mixture using an electric hydraulic press.

roacétique à $12 \%$. Les acides aminés totaux étaient dosés sur les phases aqueuses après hydrolyse par l'acide chlorhydrique $5,7 \mathrm{~N}$ à $110^{\circ} \mathrm{C}$ pendant $24 \mathrm{~h}$ et les acides aminés libres après déprotéinisation des phases aqueuses par l'acide sulfosalicilique. Le dosage des acides aminés était réalisé à l'aide de l'analyseur d'acides aminés Pharmacia LKB - Alpha Plus série 2 (Pharmacia Biotech., Saint-Quentin-Yvelines, France).

\section{RÉSULTATS ET DISCUSSION}

\section{Emmentals et rendements au pressage}

Les emmentals prélevés aux 4 stades d'affinage ont une composition globale très voisine avec des teneurs en substance sèche respectivement de $632,637,633$ et $635 \mathrm{~g} / \mathrm{kg}$ pour EmA, EmB, EmC et EmD. Les 4 fromages ont une teneur en matière grasse de $295 \mathrm{~g} / \mathrm{kg}$. Les teneurs en cendres totales sont respectivement de 32,$8 ; 32,9 ; 33,5$ et $33,2 \mathrm{~g} / \mathrm{kg}$ pour $\mathrm{EmA}, \mathrm{EmB}, \mathrm{EmC}$ et EmD.

Les cinétiques d'écoulement moyennes de la phase aqueuse et de l'huile au cours du pressage sont présentées sur la figure 1. Le rendement en huile est en moyenne de $12,1 \%$ mais varie de 9,8 à $15 \%$. Le rendement moyen en phase aqueuse à l'issue du pressage est de $8,9 \%$, soit $160 \mathrm{~g}$ obtenus pour $1,8 \mathrm{~kg}$ d'emmental pressé. Ce rendement est variable et dépend du degré d'affinage : élevé en sortie de saumure $(9,9 \pm$ $0,5 \%$ sur $E m A)$, faible en entrée de cave chaude $(7,5 \pm 0,2 \%$ sur $E m B)$ et moyen en sortie de cave chaude et sur emmental affiné $(9,0 \pm 0,2 \%$ sur $\mathrm{EmC}$ et $8,9 \pm 0,6 \%$ sur EmD).

\section{Numérations bactériennes}

Dans 11 des 12 phases aqueuses, aucun coliforme n'a pu être dénombré sur $1 \mathrm{ml}$ de phase aqueuse. Seule une des 3 phases $\mathrm{A}$ a présenté 11 coliformes $/ \mathrm{ml}$, probablement à cause d'une contamination accidentelle de la portion de fromage soumise à l'extraction.

La flore mésophile aérobie totale augmente principalement en cave froide (tableau I), puis se maintient au cours de l'affinage. Les colonies apparaissent, sur milieu PCA, blanches, à bords lisses, de diamètre 0,3 à $1 \mathrm{~mm}$. L'examen microscopique montre que ces colonies sont formées de coccobacilles isolés ou surtout en chaînettes tortueuses. Cette flore n'a pas été identifiée.

Les bactéries propioniques sont peu nombreuses dans la phase aqueuse en tout début d'affinage $\left(10^{3} \mathrm{ufc} / \mathrm{g}\right.$ de phase $\left.A\right)$, elles se développent peu pendant la période en cave froide $\left(1,6.10^{4} \mathrm{ufc} / \mathrm{g} \mathrm{B}\right)$ puis fortement en cave chaude $\left(2,5.10^{7} \mathrm{ufc} / \mathrm{g} \mathrm{C}\right)$ pour atteindre $2,5.10^{8}$ ufc/g de phase aqueuse D. Cette 
Tableau I. Flore (log ufc/ml), pH et composition $(\mathrm{g} / \mathrm{kg}$ ) des phases aqueuses d'emmental à 4 stades de l'affinage.

Flora (log cfu/ml), pH and composition $(\mathrm{g} / \mathrm{kg})$ of Emmental aqueous phase at 4 stages of ripening.

\begin{tabular}{lcccc}
\hline & A & B & C & D \\
\hline Flore méso-aérobie & 5,3 & 7,4 & 7,3 & 7,7 \\
Propionibactéries & 3,0 & 4,2 & 7,4 & 8,4 \\
pH & 5,4 & 5,4 & 5,7 & 5,8 \\
Extrait sec & 89,6 & 96,4 & 159,4 & 169,5 \\
Cendres totales & 33,4 & 30,8 & 39,5 & 34,3 \\
P & 0,96 & 0,92 & 1,35 & 1,45 \\
Ca & 7,01 & 7,55 & 7,37 & 7,89 \\
Mg & 0,63 & 0,64 & 0,68 & 0,66 \\
K & 2,56 & 2,50 & 2,46 & 2,34 \\
Na & 6,35 & 5,07 & 8,77 & 6,00 \\
I & 7,59 & 6,94 & 11,38 & 7,65 \\
Acétate & 0,0 & 0,0 & 5,8 & 5,0 \\
Propionate & 0,0 & 0,0 & 4,9 & 4,1 \\
Butyrate & 4,1 & 4,6 & 6,5 & 5,6 \\
Citrate & 3,7 & 3,7 & 0,0 & 0,0 \\
Lactate & 42,4 & 39,5 & 29,9 & 31,8 \\
lactate (L+) & 28,6 & 23,8 & 14,1 & 14,5 \\
NT (N x 6,38) & 20,4 & 28,6 & 86,7 & 99,6 \\
NCN (N x 6,38) & 20,0 & 27,6 & 80,9 & 91,0 \\
NPN (N x 6,38) & 10,4 & 15,3 & 60,0 & 73,6 \\
\hline
\end{tabular}

A, B, C, D : voir texte. See text.

évolution est conforme aux cinétiques de croissance décrites au sein du fromage (Hettinga et al, 1974 ; Fryer et Peberdy, 1977 ; Gilles et al, 1983 ; Baer et al, 1993).

\section{pH, extrait sec, sucres et acides}

L'évolution du pH dans la phase aqueuse (tableau I) est similaire à celle décrite par Lucey et al (1993).

La teneur en substance sèche de la phase aqueuse du fromage emmental sortie saumure (A) est de $89,6 \mathrm{~g} / \mathrm{kg}$. Une telle valeur est très supérieure à celle habituellement déterminée pour le lactosérum $(65 \mathrm{~g} / \mathrm{kg}$ ) (Pearce, 1992). L'origine de l'accroissement se trouve dans la forte teneur en cendres
(33,4 g/ $\mathrm{kg}$ contre $5,3 \mathrm{~g} / \mathrm{kg}$ ) conséquence de l'acidification sous presse et du saumurage du fromage. Bien que les phases $B, C$ et $D$ soient issues de fabrications fromagères différentes, il est possible, en première approche, de relier les accroissements de leurs teneurs en substance sèche à la protéolyse du réseau caséique ; la teneur en NT déjà très élevée en $A(20,4 \mathrm{~g} / \mathrm{kg}$, contre $7-8 \mathrm{~g} / \mathrm{kg}$ dans un lactosérum) est pratiquement multipliée par 5 dans D. En supposant que toute l'eau contenue dans le fromage EmD soit solvante, il peut être calculé que 169,5 x $365 / 830,5=74,5 \mathrm{~g}$ de la matière sèche de EmD par kg de fromage sont solubilisés dans la phase aqueuse ce qui représente $22 \%$ de la substance sèche non grasse. 
Aucun sucre résiduel : lactose, glucose, galactose, n'a été trouvé dans les phases $A$, $B, C$ et $D$. La teneur en lactate de la phase $A$ correspond à $90 \%$ du lactose, contenu dans l'eau solvante du lait, ce qui est usuel en fromage à pâte pressée cuite (Steffen et al, 1993). L'ion lactate est majoritairement présent sous la forme $L$ dans $A(67 \%)$ et dans B $(60 \%)$. En revanche, cette forme $L$ devient légèrement minoritaire, une fois la fermentation propionique réalisée, autour de $46-47 \%$ de lactate $L$ dans les phases $C$ et $D$. Les teneurs en lactate résiduel $(29,9 \mathrm{~g} / \mathrm{kg}$ dans $\mathrm{C}$ et $31,8 \mathrm{~g} / \mathrm{kg}$ dans D) correspondent respectivement à des teneurs théoriques de $29,9 \times 0,367 / 0,8406=$ $13 \mathrm{~g} / \mathrm{kg}$ de fromage $\mathrm{EmC}$ et $31,8 \times 0,365 /$ $0,8305=14 \mathrm{~g} / \mathrm{kg}$ de fromage EmD. De telles valeurs sont nettement supérieures à celles décrites (1,6 à $6,7 \mathrm{~g} / \mathrm{kg}$ ) par Favier et al (1984) et Feinberg et al (1987).

La teneur en acide propionique de la phase $C$ est de $66,2 \mathrm{mmol} / \mathrm{kg}$ ou $78,7 \mathrm{mmol} / \mathrm{kg}$ d'eau. Cette valeur est inférieure de plus de $20 \%$ à celle indiquée par Steffen et al (1993) pour la totalité du fromage âgé de 5 mois. La phase $\mathrm{C}$ ne représentant que $36,7 \%$ du poids du fromage, il est évident que l'acide propionique produit au cours de la fermentation propionique doit être également solubilisé dans la phase grasse du fromage. Selon Dunn et al (1986) le coefficient de partage de l'acide propionique entre le 1-octanol et l'eau est de 2,14; en assimilant la matière grasse laitière au 1-octanol (Dumont, 1994 ; communication personnelle), il peut être calculé que la teneur en acide propionique du fromage $\mathrm{C}$ devrait être égale à $(78,7 \times 0,367)+$ $(78,7 \times 0,295 \times 2,14)$ soit $78,6 \mathrm{mmol}$ par $\mathrm{kg}$ de fromage. Cette dernière valeur est voisine de celles habituellement rencontrées dans les fromages emmental de l'Ouest de la France. Il aurait été intéressant de la comparer avec la valeur trouvée sur l'EmC mais cette détermination n'a pas été réalisée dans le cadre de cette étude. La valeur déterminée dans la phase $\mathrm{D}$ inférieure à celle de $\mathrm{C}$ doit probablement correspondre à une conduite différente de l'affinage de l'EmD par rapport à celle de l'affinage de l'EmC.

Le rapport propionate/acétate est égal à 0,84 dans la phase $C$ et à 0,82 dans la phase D. Les teneurs en acétate des phases aqueuses des fromages sont à relier tant aux propriétés fermentaires caractéristiques des souches de Propionibacterium utilisées comme levains qu'à la faible solubilité dans la matière grasse laitière de l'ion acétate (coefficient 1-octanol/eau $=0,676$ ) selon Dunn et al (1986).

\section{Minéraux}

Lors de la transformation du lait en fromage emmental, la très grande majorité du lactosérum (98 à $99 \%$; ITG, 1986) est séparée des grains de caillé avant que ne se réalise l'acidification. II en découle que le relargage des minéraux liés aux caséines sous l'effet de l'abaissement du $\mathrm{pH}$ est réalisé pratiquement dans la seule phase aqueuse du caillé soumis à l'opération de pressage. La teneur en caséine du fromage emmental étant approximativement 10 fois celle du lait, le taux d'accroissement des ions minéraux dans la phase aqueuse pourrait être proportionnel à ce facteur de concentration dans la limite des solubilités maximales.

La teneur en citrate des phases $A$ et $B$ est de $3,7 \mathrm{~g} / \mathrm{kg}$, soit le double de la teneur habituelle du lait : $1,8 \mathrm{~g} / \mathrm{kg}$ (Alais, 1984). Cet accroissement trouve son origine dans le relargage total du citrate de calcium colloïdal : $0,18 \mathrm{~g} / \mathrm{kg}$ (Alais, 1984) selon le mécanisme évoqué ci-dessus. Le citrate est, par la suite, entièrement métabolisé lors de l'affinage en cave chaude.

La teneur en calcium des phases $A$ et $D$ représente près de 20 fois la teneur en calcium soluble du lait (à $\mathrm{pH} 6,6)$ et respectivement 13 et 8 fois celle du lait porté à $\mathrm{pH} 5,8$ et 5,4 (Brulé et al, 1974). Cependant, en supposant que toute l'eau résiduelle du fromage soit sol- 
vante, le calcium dissous dans la phase $\mathrm{C}$ ne représente que $31 \%$ du calcium total du fromage correspondant $(10,3 \mathrm{~g} / \mathrm{kg}$ déterminé dans EmC).

La teneur en phosphore des phases A, B, C et $D$ est relativement faible : 1,7 fois celle du $\mathrm{P}$ soluble du lait au même pH (Brulé et al, 1974) pour $A$ et $B$ et environ 3-4 fois celle du lait à $\mathrm{pH} 5,8$ pour les phases $\mathrm{C}$ et D. Ces faibles teneurs des phases aqueuses sont vraisemblablement le résultat de la force ionique élevée (salage - solubilisation des sels colloïdaux - protéolyse) qui en abaissant les pK retarde la solubilisation du phosphore colloïdal. Le phosphore soluble ne représente que $5,7 \%$ du $P$ total du fromage entrant en cave chaude (teneur en $P$ de $E m B: 6,48 \mathrm{~g} / \mathrm{kg}$ ) et $9,0 \%$ du fromage sortant de la cave chaude (teneur en $\mathrm{P}$ de $\mathrm{EmC}: 6,56 \mathrm{~g} / \mathrm{kg}$ ). Les rapports $\mathrm{Ca} / \mathrm{P}$ dans les phases $\mathrm{A}, \mathrm{B}, \mathrm{C}$ et $\mathrm{D}$ sont respectivement de 7,$3 ; 8,2 ; 5,4$ et 5,4 , soit des valeurs très éloignées de celles déterminées pour le fromage, 1,57 dans EmC. Ces résultats diffèrent de ceux de Lucey et al (1993) qui indiquent des valeurs de $\mathrm{Ca} / \mathrm{P}$ pour des phases équivalentes à $B, C$ et $D$ de 2,$8 ; 2,7$ et 2,1 et de 1,$45 ; 1,42$ et 1,07 pour les fromages correspondants. De telles différences résultent probablement de la mise en oeuvre de technologies de fabrication et d'affinage différentes : $\mathrm{pH}$ de l'emmental en entrée de cave chaude de 5,2 dans l'étude de Lucey et al. II n'en reste pas moins que les équilibres phosphocalciques de la phase aqueuse du fromage sont profondément modifiés par rapport à ceux existant dans le lait mis en oeuvre. La nature des formes salines présentes aux différents stades d'affinage reste à déterminer.

Les teneurs en sodium et en chlorure varient significativement selon les échantillons indiquant des saumurages différents selon les fabrications. Sur un autre plan, compte-tenu que les ions $\mathrm{Na}$ et $\mathrm{Cl}$ proviennent dans leur très grande majorité du sel "pris" par les fromages lors de ce saumurage, le rapport
$\mathrm{Cl} / \mathrm{Na}$ devrait être proche de celui existant dans la molécule, soit 1,54, or il est de 1,20; 1,$37 ; 1,30$ et 1,27 respectivement dans les phases $A, B, C$ et $D$ soit des valeurs de 11 à $22 \%$ plus faibles que la valeur théorique. II est fort possible que l'ion Cl soit mobilisé pour former avec les acides aminés libres des chlorhydrates ou d'autres formes moléculaires complexes avec les acides organiques et ne soit pas dosé, n'étant pas sous forme libre. Favier et al (1984) soulignent avoir répertorié dans la littérature des rapports $\mathrm{Cl} / \mathrm{Na}$ variant entre 0,82 et 2,56 .

La teneur en potassium des phases aqueuses varie entre 2,34 et $2,56 \mathrm{~g} / \mathrm{kg}$ soit des valeurs supérieures à celle déterminée dans le lait. Comme pour l'ion citrate, cet accroissement trouve son origine dans le relargage, au cours de l'acidification sous presse du potassium fixé aux micelles de caséine : $75 \mathrm{mg} / \mathrm{kg}$ de lait selon Le Graët et Brulé (1993) à pH 5,4. Les valeurs déterminées pour le magnésium résultent du même mécanisme.

Les teneurs en $\mathrm{Cu}, \mathrm{Mn}$ et $\mathrm{Fe}$ ont également été déterminées dans les phases $A, B$, $C$ et $D$. Les valeurs de Cu et $M n$, respectivement égales à $0,6 \mathrm{mg} / \mathrm{kg}$ et $0,4 \mathrm{mg} / \mathrm{kg}$ ne varient pas au cours de l'affinage. Les teneurs en Fe étaient respectivement de 5,$6 ; 3,7 ; 1,5$ et $2,1 \mathrm{mg} / \mathrm{kg}$ dans $\mathrm{A}, \mathrm{B}, \mathrm{C}$ et $\mathrm{D}$. La décroissance en Fe constatée peut trouver son origine soit dans une variation des teneurs des laits de fabrication, soit dans l'utilisation de cet élément par les micro-organismes du fromage.

\section{Matières azotées}

L'affinage en cave chaude multiplie par près de 5 la valeur de la matiere azotée totale qui atteint une valeur proche de $100 \mathrm{~g} / \mathrm{kg}$ en fin d'affinage. Les matières azotées présentes dans les phases $A$ et $B$ sont pratiquement constituées à $100 \%$ de formes solubles à $\mathrm{pH} 4,6$ dont près de la moitié so- 
lubles dansl'acidetrichloroacétique(TCA)à $12 \%$ : les rapports NCN/NT et NPN/NT des phases $A, B, C$ et $D$ sont respectivement égaux à 0,98 et 0,$51 ; 0,96$ et 0,$53 ; 0,93$ et 0,$69 ; 0,91$ et 0,74 . Ces valeurs indiquent que si l'essentiel de la protéolyse conduit à des formes solubles dans le TCA $12 \%$ (multiplication par 7 des teneurs entre $A$ et $D$ ) il y a aussi libération de gros fragments de caséine insolubles à pH 4,6 (environ $10 \%$ des matières azotées totales de la phase $D$ ). Les valeurs déterminées pour les matières azo- tées totales sont en bon accord avec celles de Lucey et al (1993).

L'examen du tableau II dans lequel sont rassemblées les teneurs en acides aminés libres et totaux montre qu'une large part des produits issus de la protéolyse est sous forme d'acides aminés libres $(26 \%$ dans A ; $56 \%$, soit $55,8 \mathrm{~g} / \mathrm{kg}$ dans D). L'accroissement des teneurs est général pour tous les acides aminés, sauf pour l'arginine qui apparaît totalement métabolisée lors de la fermentation propionique. Cet acide aminé

Tableau II. Composition en acides aminés totaux en $\mathrm{g} / \mathrm{kg}$ (\% sous la forme libre) des phases aqueuses d'emmental à 4 stades de l'affinage.

Total amino-acids composition in $\mathrm{g} / \mathrm{kg}$ (free amino-acid \%) of Emmental aqueous phase at 4 stages of ripening.

\begin{tabular}{lcrrr}
\hline Acides aminés & $A$ & $B$ & $C$ & $D$ \\
\hline Total (\% libre) & $20,1(26)$ & $27,6(50)$ & $84,6(50)$ & $99,6(56)$ \\
Asp & $1,3(4)$ & $4,5(\mathrm{~h} 7)$ & $4,5(7)$ & $6,2(13)$ \\
Thr & $0,9(18)$ & $1,1(21)$ & $3,0(45)$ & $3,3(55)$ \\
Ser & $1,2(12)$ & $1,7(12)$ & $4,5(23)$ & $5,1(31)$ \\
Glu & $4,9(20)$ & $6,8(27)$ & $22,6(38)$ & $27,0(48)$ \\
Pro & $1,8(45)$ & $2,2(42)$ & $7,5(67)$ & $8,7(86)$ \\
Gly & $0,4(23)$ & $0,6(37)$ & $1,7(64)$ & $1,9(80)$ \\
Ala & $1,0(55)$ & $1,2(59)$ & $2,6(70)$ & $2,9(73)$ \\
Val & $1,1(27)$ & $1,5(40)$ & $5,0(80)$ & $6,5(71)$ \\
Met & $0,3(40)$ & $0,3(64)$ & $1,3(76)$ & $1,6(68)$ \\
Ile & $1,0(14)$ & $1,4(18)$ & $4,4(47)$ & $5,0(54)$ \\
Leu & $1,6(27)$ & $2,1(49)$ & $7,2(76)$ & $8,4(82)$ \\
Tyr & $0,4(21)$ & $0,5(34)$ & $1,5(55)$ & $1,7(58)$ \\
Phe & $0,7(19)$ & $0,8(29)$ & $2,5(64)$ & $3,0(76)$ \\
His & $0,5(34)$ & $0,7(31)$ & $1,8(40)$ & $1,3(23)$ \\
Lys & $2,0(28)$ & $2,7(39)$ & $10,7(56)$ & $13,2(54)$ \\
Arg & $0,8(48)$ & $1,1(40)$ & $1,3(0)$ & $1,3(0)$ \\
Cys & 0,1 & 0,1 & 0,2 & 0,4
\end{tabular}

Acides aminés libres $(\mathrm{g} / \mathrm{kg})$

\begin{tabular}{lllll} 
PSer & 0,2 & 0,3 & 1,0 & 1,1 \\
Asn & 0,3 & 0,6 & 1,4 & 4,2 \\
Gin & 0,2 & 0,4 & 2,4 & 4,3 \\
Orn & 0,0 & 0,1 & 1,4 & 2,6 \\
Cit & 0,0 & 0,0 & 1,0 & 0,9 \\
\hline
\end{tabular}


est-il limitant pour les bactéries propioniques? Aucune donnée n'étant disponible à la connaissance des auteurs, cette interrogation reste entière et requiert un approfondissement des connaissances sur le métabolisme azoté de ce genre bactérien.

La teneur en proline libre, acide aminé considéré comme intervenant dans la saveur de l'emmental (Langsrud et Reinbold, 1973) atteint $7,5 \mathrm{~g} / \mathrm{kg}$ dans D. Au moins 2 acides aminés (Glu et Tyr) apparaissent en sursaturation par rapport à leur solubilité maximale dans l'eau à $25^{\circ} \mathrm{C}$ : respectivement 8,58 et $0,454 \mathrm{~g} / \mathrm{kg}$ selon Hutchens et Kirby Hade (1968). L'accroissement de solubilisation constaté, respectivement $51 \%$ et $117 \%$, trouve probablement son origine dans la force ionique élevée des phases aqueuses.

\section{CONCLUSION}

Les résultats obtenus au cours de cette étude confirment l'intérêt de l'extraction et de la caractérisation de la phase aqueuse du fromage emmental au cours de l'affinage. La composition de ce liquide interne traduit complètement les effets des paramètres de fabrication: stade technologique et cinétique de l'acidification par exemple, et le métabolisme des bactéries lactiques et de celles d'affinage sous l'effet des conditions (temps, température et hygrométrie) dans lesquelles sont placés les fromages. Son étude constitue donc une méthodologie privilégiée pour déterminer de manière approfondie les effets de telle ou telle modification technologique, de tel ou tel écosystème bactérien ajouté au lait de fabrication ou de telle ou telle conduite de l'affinage.

Ces effets seront d'autant mieux appréhendés que l'analyse portera sur des phases aqueuses extraites de fromages issus de la même fabrication. Tel n'était pas le cas de la présente étude. Ce sera l'objet d'un prochain travail incluant d'autres stades de maturation.

La méthodologie proposée pour l'emmental est aisément transposable à toutes les variétés de fromage à pâte dure et probablement sous réserves de modifications du protocole d'extraction aux variétés de fromage à pâte molle. Elle ouvre donc un nouveau et vaste champ d'investigations sur le suivi et l'approfondissement des mécanismes de l'affinage.

\section{REMERCIEMENTS}

Nous tenons à remercier M Garnier pour son importante contribution à la réalisation de la presse prototype, ainsi que J Fauquant pour son assistance.

Ce travail a été cofinancé par l'Institut national de la recherche agronomique (AIP Matural) et la région Bretagne.

\section{RÉFÉRENCES}

Adda J (1987) Formation de la flaveur. In : Le Fromage (Eck A, Ed). Lavoisier Paris, 330-340

Alais C (1984) Science du lait : principes des techniques laitières, $4^{\ominus}$ éd. Edition Sepaic, Paris

Baer A, Ryba I, Grand M (1993) Ursachen der Entstehung von braunen Tupfen im Käse. Schweiz Milchw Forschung 22, 3-7

Barthel C, Sanderg E, Haglund E (1928a) Une nouvelle méthode pour l'étude du procédé de la maturation des fromages. Lait 8, 285-288

Barthel C, Sanderg E, Haglund E (1928b) Recherches sur la présure dans les fromages. Lait 8, 762-768 et 891-897

Brulé G, Maubois JL, Fauquant J (1974) Étude de la teneur en éléments minéraux des produits obtenus lors de l'ultrafiltration du lait sur membrane. Lait 54, 600-615

Dunn WJ, Block JH, Pearlman RS (1986) Partition coefficient: determination and estimation. Pergamon Press, New York, 26

Favier JC, Ansoborio MJ, Daval L, Pradier F (1984) Composition des fromages à pâte cuite pressée. Cah Nutr Diét 19, 43-55 
Favrot C (1994) Croissance de Lactococcus lactis subsp lactis dans le lait : effet de la coagulation par la présure. Thèse, ENSA, Rennes

Feinberg M, Favier JC, Ireland-Ripert J (1987) Fromage pâte dure : emmental pressé cuit. In : Répertoire général des aliments. T2: Table de composition des produits laitiers. Lavoisier, Paris

FIL (Fédération internationale de laiterie) (1987) Détermination de la teneur en phosphore total dans les fromages. Norme $33 \mathrm{C}$

Fryer TF, Peberdy MF (1977) Growth of propionibacteria in Swiss and Egmond cheese. NZJ Dairy Sci Technol 12, 421-426

Gilles J, Turner KW, Martley FG (1983) Swisstype cheese. I.Manufacturing and sampling procedures. NZJ Dairy Sci Techno/ 18, 109-115

Hettinga DH, Reinbold GW, Vedamuthu ER (1974) Split defect of Swiss cheese. I. Effect of strain of propionibacteria and wrapping material. J Milk Food Technol 37, 322-328

Hutchens JO, Kirby Hade EP (1968) Solubilities of amino acids in water at various temperatures. In: Handbook of Biochemistry (Sober HA, Ed). The Chemical Rubber Co, Cleveland, B10

ITG (1986) Influence de la durée des brassages avant et après le chauffage sur l'acidification, l'égouttage et la qualité de l'emmental. Étude ITG zone Sud 1986/04/B, La Roche-sur-Foron, France

Langsrud T, Reinbold GW (1973) Flavor development and microbiology of Swiss cheese - a review. III. Ripening and flavor production. $J$ Milk Food Technol 36, 593-609
Le Graët Y, Brulé G (1993) Les équilibres minéraux du lait : influence du $\mathrm{pH}$ et de la force ionique. Lait $73,51-60$

Lucey JA, Gorry C, Fox PF (1993) Changes in the acid-base buffering curves during the ripening of emmental cheese. Milchwissenschaft 48, $183-186$

Madec MN, Rouault A, Maubois JL, Thierry A (1994) Milieu sélectif pour le dénombrement des bactéries propioniques. Brevet européen $n^{\circ}$ dépot PCT/FR94 00082

Maubois JL, Mocquot G (1971) Préparation de fromage à partir de "pré-fromage liquide " obtenu par ultrafiltration du lait. Lait 51, 495-533

McDowall FH, Dolby RM (1936) Studies on the chemistry of Cheddar cheese making. J Dairy Res 7, 156-175

Morris MA, Holt C, Brooker BE, Banks JM, Manson W (1988) Inorganic constituents of cheese : analysis of juice from a one-month-old cheddar cheese and the use of light and electron microscopy to characterize the crystalline phases. J Dairy Res 55, 255-268

Pearce RJ (1992) Whey processing. In : Whey and Lactose Processing (Zadow JG, ed). Elsevier, London, 73-89

Sandberg E, Haglund E, Barthel C (1930) L'analyse du jus de fromage comme moyen de déterminer le degré de la maturation. Lait 10, 1-21

Steffen C, Eberhard P, Bosset JO, Rüegg M (1993) Swiss-type varieties. In: Cheese : chemistry, physics and microbiology. Major cheese groups, vol 2 (Fox PF ed). Elsevier, London, 83-110 